\title{
FATORES ASSOCIADOS AO CONTROLE GLICÊMICO EM IDOSOS DIABÉTICOS NÃO INSTITUCIONALIZADOS
}

Leiliane Moraes dos Santos Silva; Universidade Federal de Pernambuco-UFPE; leiliane.moraes@ufpe.br Crislayne Maria Berto; Universidade Federal de Pernambuco-UFPE; crislayneberto@gmail.com Fernando Arthur Alves da Silva; Universidade Federal de Pernambuco-UFPE; fernando.arthur@ufpe.br Kimberly Mayara Gouveia Bezerra; Universidade Federal de Pernambuco-UFPE; kimberly.gouveia@ufpe.br

Ana Paula de Oliveira Marques; Universidade Federal de Pernambuco-UFPE; ana.marques@ufpe.br

\section{RESUMO}

Introdução: Nos últimos anos houve um aumento do número de longevos no Brasil. Com a mudança no perfil epidemiológico do país, registra-se um aumento do número de doenças não transmissíveis (DNT), como a obesidade e a hipertensão. O descontrole glicêmico e hábitos de vida indesejáveis implicam no surgimento dessas doenças. Na velhice, o Diabetes Mellitus (DM) se associa ao aumento da mortalidade e na diminuição da qualidade de vida. Assim, é necessário estudar a correlação das DNTs supracitadas visando minimizar seus efeitos durante o envelhecimento. Objetivo: Revisar artigos que discutem os fatores associados ao controle glicêmico adequado em idosos não institucionalizados. Métodos: Revisão integrativa, com recorte temporal de 10 anos, nas plataformas de pesquisa PubMed, Scielo, e Lilacs, utilizando a combinação dos descritores em português "Diabetes", "Idoso" e "Glicemia". Foram encontrados 323 estudos. Após a leitura, 318 estudos foram excluídos por não abordarem a temática, indisponibilidade na íntegra e por repetição, sendo selecionados 40 estudos. Resultados: Os fatores biológicos, socioeconômicos, patológicos e temporais são elementos significativos no controle glicêmico eficaz. Há uma necessidade de monitorização regular dos pacientes com DM e a criação de um plano de cuidado individualizado. Concomitante a isso, os exercícios físicos e uma alimentação saudável proporcionará um bem-estar íntegro, afinal, esses hábitos agem positivamente, principalmente quando empregados coletivamente. Conclusão: $\mathrm{O}$ conhecimento dos fatores que se relacionam com o controle glicêmico eficaz e sua atuação como barreira ou facilitador da terapêutica acrescenta positivamente na elaboração de linhas de cuidado individualizados, melhorando a qualidade de vida dos longevos.

Palavras-chave: Diabetes; Idoso; Glicemia. 